Pathologe $2020 \cdot 41: 39-45$

https://doi.org/10.1007/s00292-019-00733-3

Online publiziert: 13. Januar 2020

(c) Der/die Autor(en) 2020

Schwerpunktherausgeber

R. Büttner, Köln

S. Schäfer, Friedrichshafen

\section{Molekularpathologische Diagnostik an zytologischen Präparaten}

Für Patienten, die mit einem fortgeschrittenen Lungenkarzinom diagnostiziert werden, ist eine rasche molekularpathologische Diagnostik erforderlich. Es gibt zugelassene Medikamente für Patienten mit EGFR- oder BRAF-Mutationen bzw. $A L K$-Translokationen [1-3], die auch in der Erstlinientherapie eingesetzt werden können. Man sucht hierbei nach unterschiedlichen genetischen Veränderungen:

1. Mutationen, z.B. Punktmutationen, Deletionen, Insertionen, also kurzstreckige Veränderungen in der Basenabfolge. Hierzu ist eine Sequenzierung mehrerer Gene oder Genabschnitte nötig (Parallelsequenzierung). Typische Beispiele sind unterschiedliche Mutationen im EGFR- oder im BRAF-Gen.

2. Translokationen, also Umlagerungen größerer Chromosomenabschnitte. Diese werden sehr gut in der Fluoreszenz-in-situ-Hybridiserungs(FISH)Analyse erkannt. Typische Bespiele sind EML4/ALK-Translokation und ROS1-Translokation $[4,5]$.

3. Amplifikationen, also Vervielfältigungen größerer Chromosomenabschnitte. Auch diese sind sehr gut in der FISH-Analyse zu erkennen. Typische Beispiele sind MET- [6] und HER2-Amplifikation [7].

Eine ALK- oder ROS1-Translokation kann immunzytologisch oder immunhistologisch mit großer Sicherheit vorausgesagt werden. Bei positiver Immun-

Jana Fassunke - Markus Ball · Marianne Engels

Institut für Pathologie, Universitätsklinikum Köln, Köln, Deutschland

\title{
Molekularpathologische Diagnostik an zytologischen Präparaten
}

zytologie/Immunhistologie für ALKoder ROS1 schließen wir in unserer Institution die FISH-Analyse zur Bestätigung an. Generell wird zusätzlich an der Probe untersucht, ob eine MET-Amplifikation oder eine RET-Translokation vorliegt. Um Resistenzmechanismen nach einer Tyrosinkinaseinhibitortherapie $\mathrm{zu}$ bestimmen, prüfen wir auf MET- oder HER2-Amplifikation. Die Frage nach einer NTRK(neurotrophe Gene der Tyrosin-Rezeptorkinase)-Fusion wird mittels RNA-basierter Parallelsequenzierung abgeklärt. Diese Untersuchung wird von einem Pathologen angefordert, nachdem zuvor keine therapierbaren Zielstrukturen gefunden werden konnten. Grundsätzlich wird bei jedem Patienten das am besten geeignete Probenmaterial für die molekularpathologische Untersuchung verwendet. In den meisten Fällen ist dies eine Biopsie. Falls das Biopsiematerial nicht ausreicht, wird die erforderliche Diagnostik am zytologischen Material durchgeführt $[8,9]$. Bei vielen Patienten wird die Diagnose eines nichtkleinzelligen Lungenkarzinoms sogar rein zytologisch gestellt. Die oben angeführten Untersuchungen sind alle an zytologischen Proben gut durchführbar, wenn die Probe hinreichend viele maligne Zellen enthält $[10,11]$. Diese malignen Zellen müssen zytomorphologisch gut von den benignen Zellen der Umgebung abgrenzbar sein. Alle üblichen Probensorten und alle Standardfärbungen der Zytologie sind hierfür geeignet. Immunzytologische Präparationen sind ebenfalls geeignet. Da die zytologischen Präparate häufig limitiert sind, empfiehlt es sich, nach der morphologischen Diagnosesicherung repräsentative Auf- nahmen als Bilder zu archivieren. Somit können die Proben für die molekularen Analysen weiterverwendet werden.

Wir wenden die genannten molekularpathologischen Untersuchungen routinemäßig an folgenden Probensorten an (-Abb. 1): Bronchusbürstenausstriche, transbronchiale Nadelaspiration (TBNA), Bronchiallavage, Punktionsausstriche von Lymphknotenmetastasen oder peripheren Metastasen, Pleuraerguss, Aszites, Perikarderguss [12, 13]. Die flüssigen Proben sind als Sedimentausstriche, Zytospinpräparate oder Zellblock aus Ergussflüssigkeit aufgearbeitet $[14,15]$. Bei der TBNA werden immer häufiger auch Dünnschichtpräparate benutzt. Dabei untersuchen wir regelmäßig auch extern angefertigte und gefärbte zytologische Präparate. Die Extraktion der DNA aus Standardfärbungen wie HE, Pappenheim oder Papanicolaou kann problemlos durchgeführt und für anschließende molekularpathologische Untersuchungen genutzt werden. Die DNA-Qualität von alkoholfixierten oder luftgetrockneten zytologische Präparationen eignet sich sehr gut für molekulargenetische Analysen, da es zu keinem formalinbedingten „crosslinking“ wie im Gewebematerial kommt.

\section{Präanalytik von zytologischen Präparaten}

Zytologische Präparate können luftgetrocknet (nicht eingedeckelt) oder eingedeckelt (Deckgläschen oder Folie) und bereits schon gefärbt zur Analyse eingesandt werden. In beiden Fällen muss ein Zytopathologe die geeigneten zu verwendenden Areale einzeichnen. Bei dem 


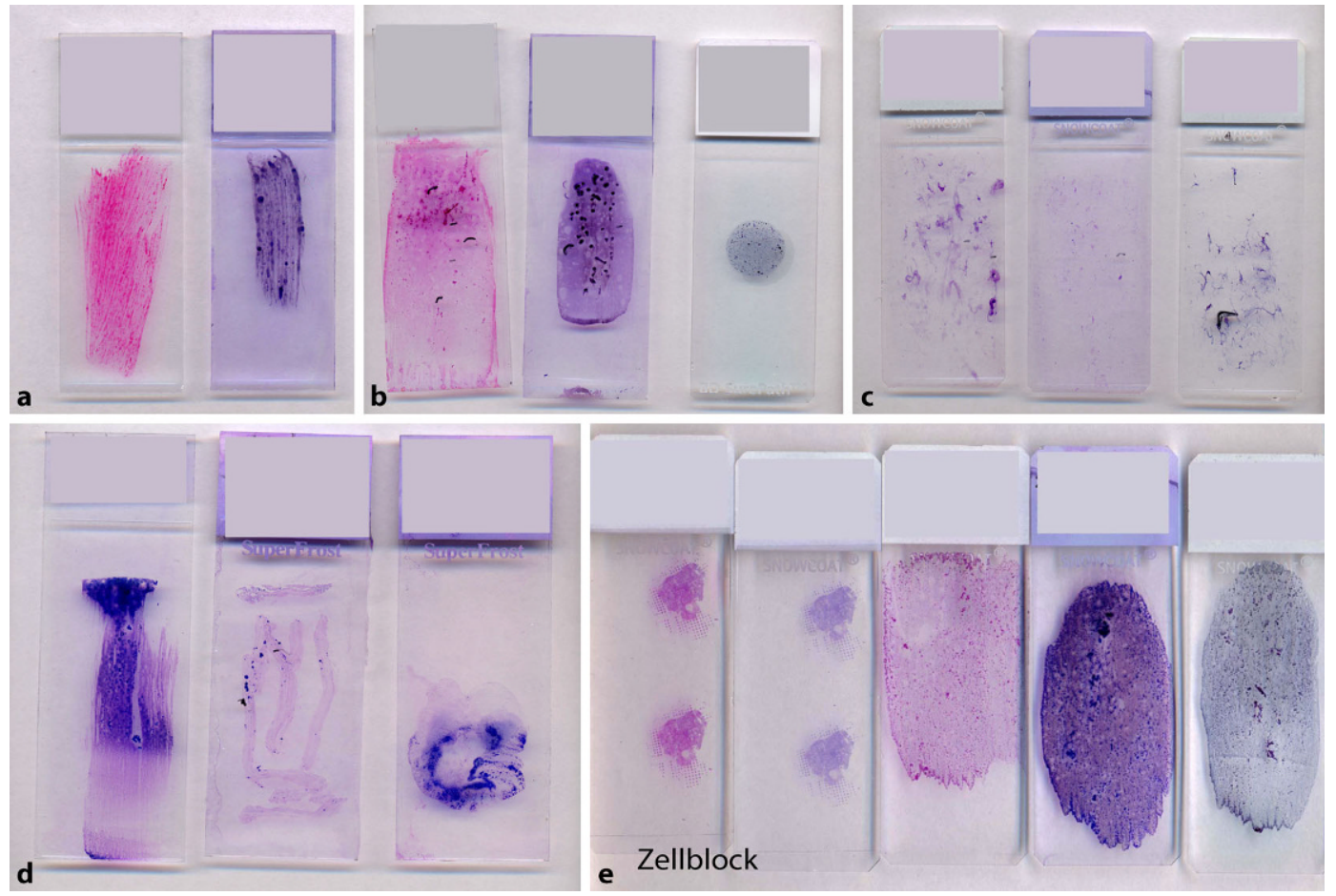

Abb. $1<$ Zytologische Präparate für die molekularpathologische Diagnostik (Auswahl): a Bürstenausstriche, $\mathbf{b}$ transbronchiale Nadelaspiration (TBNA) mit Dünnschichtpräparat, c Bronchiallavage, d Lymphknotenpunktate und Lymphknotentupfpräparat, e Ergussflüssigkeit mit Zellblock. (@ M. Engels) nicht eingedeckelten Präparat werden die Areale auf der Rückseite des Objektträgers mit einem wasserfesten Stift markiert. Je nachdem wie die Zellen verteilt sind, können es ein oder mehrere Areale auf einem Objektträger sein. Ist die Markierung auf der Vorderseite (Folie/ Deckgläschen), überträgt man die Markierung auf die Rückseite des Objektträgers. Bei allen zytologischen Präparaten kratzt man mit einem Diamantstift die Markierung auf der Rückseite des Objektträgers ins Glas. Diese Markierung wird dann nochmals mit einem wasserfesten Stift nachgezogen. In den Rillen verfangen sich kleinste Farbpigmente, die vom Xylol nicht weggewaschen werden (- Abb. 2a-c). Diese Farbspuren und die Spuren des Diamantstiftes erleichtern es, den markierten Bereich wiederzufinden.

Ein luftgetrockneter Schnitt wird für eine DNA-Extraktion für eine Minute in Wasser gehalten. Anschließend kann mittels Makrodissektion mit einem Skalpell das markierte Areal abgekratzt werden. Das dissezierte Material wird mit der Skalpellspitze in ein Reaktionsgefäß, in dem sich Lysepuffer befindet, überführt. Das Gemisch wird über Nacht bei $70^{\circ} \mathrm{C}$ geschüttelt und je nach Herstellerangaben weiterverarbeitet. Die extrahierte DNA steht für alle PCR(Polymerase-
Kettenreaktion)-basierten Untersuchungen zur Verfügung.

Sind die eingesandten Proben eingedeckelt oder mit Folie überzogen, muss zuerst das Deckglas bzw. die Folie entfernt werden. Ein Präparat, das mit einem Deckgläschen eingedeckelt ist, wird über Nacht in Xylol gestellt. Lässt sich am nächsten Morgen das Deckgläschen nicht entfernen, wird der Schnitt für $30 \mathrm{~min}$ in einen $-80^{\circ} \mathrm{C}$ kalten Gefrierschrank gelegt. Anschließend wird der Schnitt wieder in Xylol gestellt, das Deckgläschen sollte nun zu entfernen sein [16]. Sind die eingesandten Proben mit Folie überzogen, werden sie für $3,5 \mathrm{~min}$ in Aceton gestellt, für $1 \mathrm{~min}$ in ein Aceton-Xylol-Gemisch (1:2) und erneut $1 \mathrm{~min}$ in Xylol, um das Eindeckmedium vollständig zu entfernen. Hier sei angemerkt, dass selbst Markierungen mit xylolfesten Stiften nicht immer haften bleiben. Der markierte Bereich des Präparats wird dann mit einem Skalpell „abgekratzt“ und in ein mit Lysepuffer befülltes Reaktionsgefäß gegeben. Mit der extrahierten DNA können alle PCR-basierten Methoden durchgeführt werden.

\section{Vorbereitung für die FISH-Analyse}

Die zytologischen Präparate müssen für die FISH-Analyse entdeckelt und auf der Rückseite mit einem Diamantstift markiert sein. Für die FISH-Analyse werden möglichst kleine Areale mit mindestens 100 Tumorzellen, die nicht überlappend liegen, markiert. Falls unterschiedliche FISH-Analysen auf einem Schnitt durchgeführt werden müssen, sollte der Abstand zwischen den Arealen möglichst groß gewählt werden. Die Vorbehandlung der Schnitte erfolgt automatisiert mit dem VP2000 der Firma Abbott (Abbott Park, USA) und ist identisch mit der Vorbehandlung von formalinfixierten, paraffineingebetteten (FFPE) Schnitten. Präparate, die bereits mit HE, Pappenheim oder Papanicolau gefärbt sind, sind für eine FISH-Analyse gut geeignet. Diese Präparate werden durch die Vorbehandlung der Schnitte für die Hybridisierung entfärbt. Nach der Vorbehandlung wird die 1 . Sonde $(3 \mu \mathrm{l})$ aufgetragen, mit einem runden Deckglas (13 mm) und Eindeckmedium eingedeckelt. Mit Fixogum wird das Deckglas verschlossen. Falls die gewünschten Areale eng beieinander liegen, können Deckgläschen geteilt werden. Nach Versiegelung des 1. Areals mit Fixogum wird die 2. Sonde 


\section{J. Fassunke $\cdot$ M. Ball $\cdot$ M. Engels}

\section{Molekularpathologische Diagnostik an zytologischen Präparaten}

\section{Zusammenfassung}

Für Lungenkarzinome mit bestimmten molekulargenetischen Veränderungen des ALK-, BRAF- oder EGFR-Gens gibt es zielgerichtete Therapien, die auch in der Erstlinientherapie zugelassen sind. Oftmals steht für die molekularpathologische Testung nur ein begrenztes Probenmaterial in Form von Biopsien zur Verfügung. In einigen Fällen weisen die Biopsien nach Standardfärbungen und immunhistochemischen Färbungen keinen oder einen zu geringen Tumoranteil auf, um PCR-basierte Untersuchungen oder FISH(Fluoreszenz-in-situ-Hybridisierung)Analysen damit durchzuführen. In solchen Fällen kann auf zytologische Präparate wie Bronchusbürstenausstriche, transbronchiale Nadelaspiration (TBNA), Bronchiallavage,
Punktionsausstriche von Lymphknotenmetastasen oder peripheren Metastasen, Pleuraerguss, Aszites und Perikarderguss zurückgegriffen werden. Standardfärbungen wie HE, Pappenheim und Papanicolaou sowie immunhistologische Präparate können nach der morphologischen Analyse und Diagnosesicherung verwendet werden, um daraus DNA zu extrahieren. Ein Zytopathologe markiert hierfür die Tumorzellareale vorher auf dem Objektträger. Nur bei ausreichend hohem Tumorzellanteil ist es möglich, diese Areale zu dissezieren und DNA zu extrahieren. Um eine FISH-Analyse mit den zytologischen Präparaten durchzuführen, müssen vom Zytopathologen möglichst kleine Areale mit mehr als 100 Tumorzellen eingezeichnet werden.
Bereits gefärbte Schnitte werden vor der Hybridisierungsreaktion entfärbt. Ziel ist es, auch bei begrenztem Ausgangsmaterial eine umfangreiche Diagnostik zu erreichen und Rebiopsien zu vermeiden. In der Pathologie der Uniklinik Köln wurden im Zeitraum von 2016 bis Juli 20191711 NGS(Next Generation Sequencing)- und FISH-Analysen an zytologischen Präparaten durchgeführt. Dabei lag die Erfolgsrate der NGS-Untersuchungen mit 85,9\% leicht über der Erfolgsrate der FISHAnalysen mit 82,8\%.

Schlüsselwörter EGFR-Gen · Fluoreszenz-in-situ-Hybridisierung · Next Generation Sequencing . Lungentumoren $\cdot$ Zytodiagnostik

\section{Molecular diagnostics of cytological specimens}

\section{Abstract}

For lung carcinomas with certain molecular genetic alterations of the $A L K, B R A F$ or EGFR gene, there are targeted therapies that are also approved as first-line therapy. Often, only limited sample material from biopsies is available for molecular pathological testing. In some cases, biopsies with standard and immunohistochemical staining have no or too low tumor content to be used for PCR-based examinations or fluorescence in situ hybridization (FISH) analyses. In such cases, cytological preparations such as bronchus brush smears, transbronchial needle aspiration (TBNA), bronchial lavage, puncture smears from lymph node or peripheral metastases, pleural effusion, ascites, and pericardial effusion can be used. Standard stainings such as HE, Pappenheim, and Papanicolaou as well as immunohistological preparations can be used after morphological analysis and confirmatory diagnosis in order to extract DNA from them or to use them for FISH analysis. A cytopathologist marks the tumor cell areas on the slide beforehand. It is only possible to dissect these areas and extract DNA if the proportion of tumor cells is sufficiently high. In order to carry out a FISH analysis with the cytological preparations, the cytopathologist must draw in areas as small as possible with more than 100 tumor cells. Already stained sections are destained before the hybridization reaction. The aim is to achieve comprehensive diagnostics even with limited starting material and to avoid re-biopsies. Between 2016 and July 2019, 1711 next generation sequencing (NGS) and FISH analyses were performed on cytological preparations at the Department of Pathology of the University Hospital of Cologne. The success rate of $85.9 \%$ for NGS examinations was slightly higher than the success rate of $82.8 \%$ for FISH analyses.

\section{Keywords}

EGFR gene - Fluorescence in situ hybridization . Next generation sequencing - Lung cancer . Cytodiagnosis aufdas 2. Areal aufgetragen, mit Eindeckmedium eingedeckelt und mit Fixogum abgedichtet (• Abb. 2d-g).

\section{DNA-Extraktion aus zytologi- schen Präparaten}

Für die DNA-Extraktion und die anschließenden PCR-basierten Untersuchungen benötigt man mindestens hundert maligne Zellen, die „pur“, d.h. möglichst wenig vermengt mit benignen Epithelien und Entzündungszellen vorliegen sollten.
Der Tumorzellgehalt sollte für eine Parallelsequenzierung mindestens $10 \%$ der insgesamt enthaltenen Zellen betragen. Für andere, PCR-basierte Untersuchungen, wie z. B. die Sanger-Sequenzierung, muss der Tumorzellgehalt bei $20 \%$ liegen, da diese Methode weniger sensitiv ist. Der prozentuale Anteil der Tumorzellen sollte angegeben werden, um bei Nachweis einer Mutation, die mittels der Parallelsequenzierung detektiert wurde, eine Aussage zur Allelfrequenz machen zu können. Um die Wahrscheinlichkeit zu erhöhen, dass eine vorhandene Muta- tion auch gefunden wird, ist es sinnvoll, nicht alle Zellen eines Objektträgers zu extrahieren, sondern in der Menge der Zellen, die extrahiert werden, die Tumorzellen anzureichern. Deshalb werden alle vorhandenen Präparate gefärbt $(\mathrm{HE})$ und mikroskopisch durchgesehen. Der Zytopathologe markiert für die DNAExtraktion einen Präparateabschnitt mit einem wasserfesten Stift auf dem Objektträger, in dem größere Mengen Tumorzellen möglichst ohne Vermengung mit benignen Zellen oder Entzündungszellen gelagert sind. Eine Überlagerung 


\section{Schwerpunkt: Lunge}
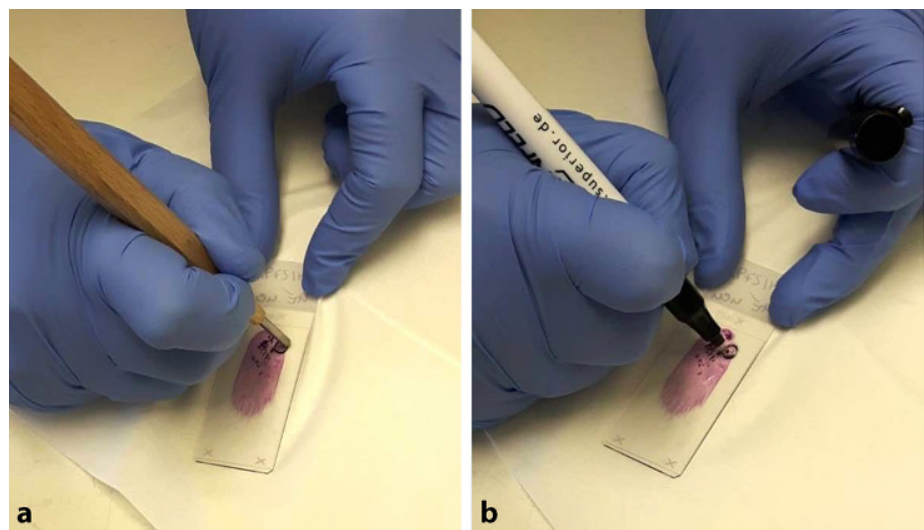

b
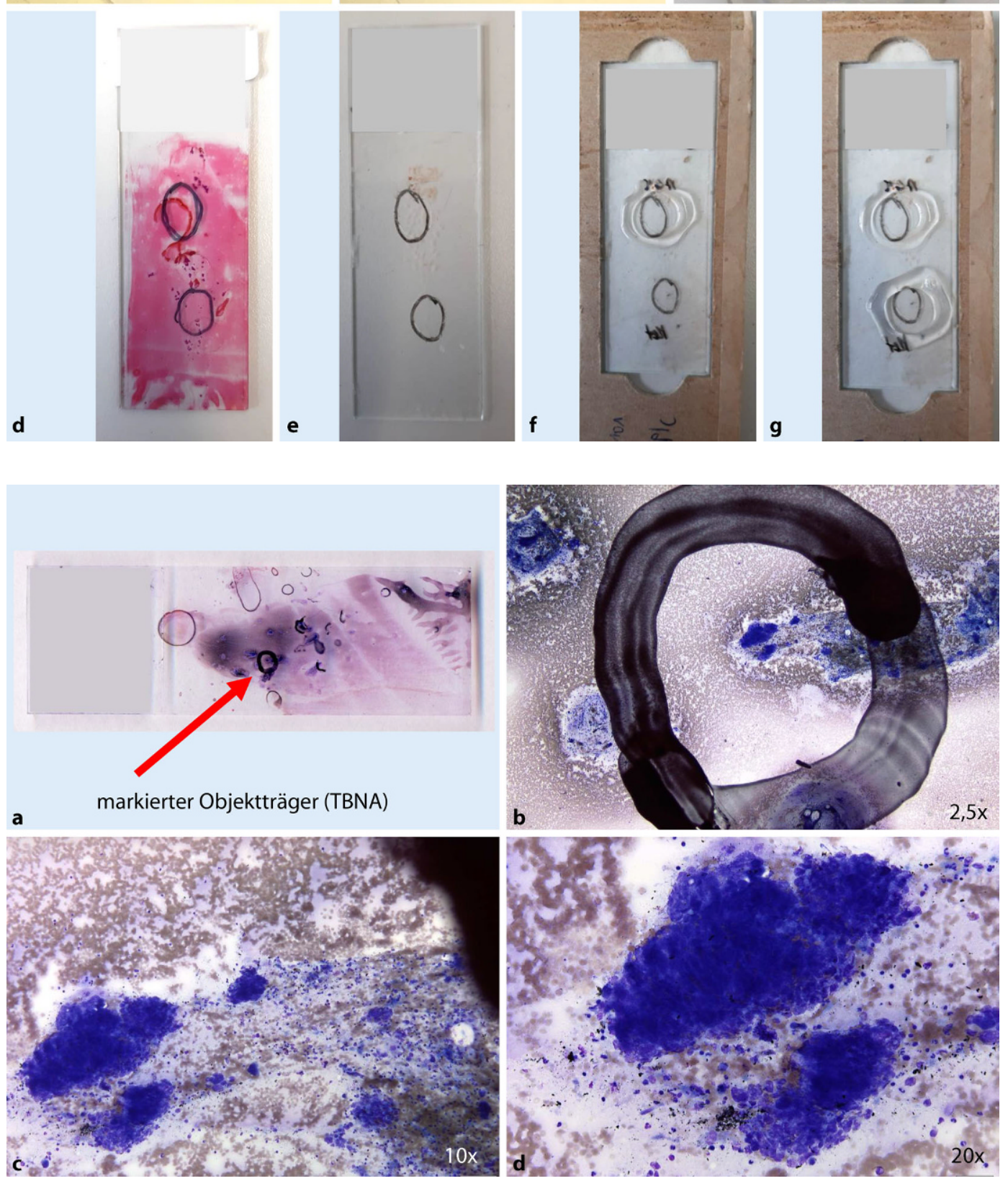

Abb. $2 \triangleleft$ Präanalytische Schritte an zytologischen Präparaten. a Verwendung eines Diamantstiftes, Einkratzen der Markierung auf der Rückseite eines Objektträgers, b Nachzeichnung mit einem wasserfesten Stift, c Entdeckeln des Objektträgers in Xylol, d geeigneter Ausstrich eines Lymphknotenpunktates (HE) mit 2 Arealen für die Fluoreszenz-in-situ-Hybridisierungs(FISH)-Analyse, e entfärbter Schnitt nach Vorbehandlung für die FISHAnalyse mit Markierung, f Auftragung der 1. Sonde mit anschließender Fixogum-Versiegelung, g Auftragung der 2. Sonde mit anschließender Fixogum-Versiegelung. (৫ M. Engels)

Abb. $3<$ a TBNA(transbronchiale Nadelaspiration)-Ausstrich mit Markierung für die DNA-Extraktion (roter Pfeil). MGG Färbung. b-d Große, dichte Tumorzellverbände mit mehr als 100 Karzinomzellen, relativ wenige Entzündungszellen in der Umgebung (b:Vergr. 2,5:1; c:Vergr. 10:1, d:Vergr. 20:1). (৫ M. Engels) 

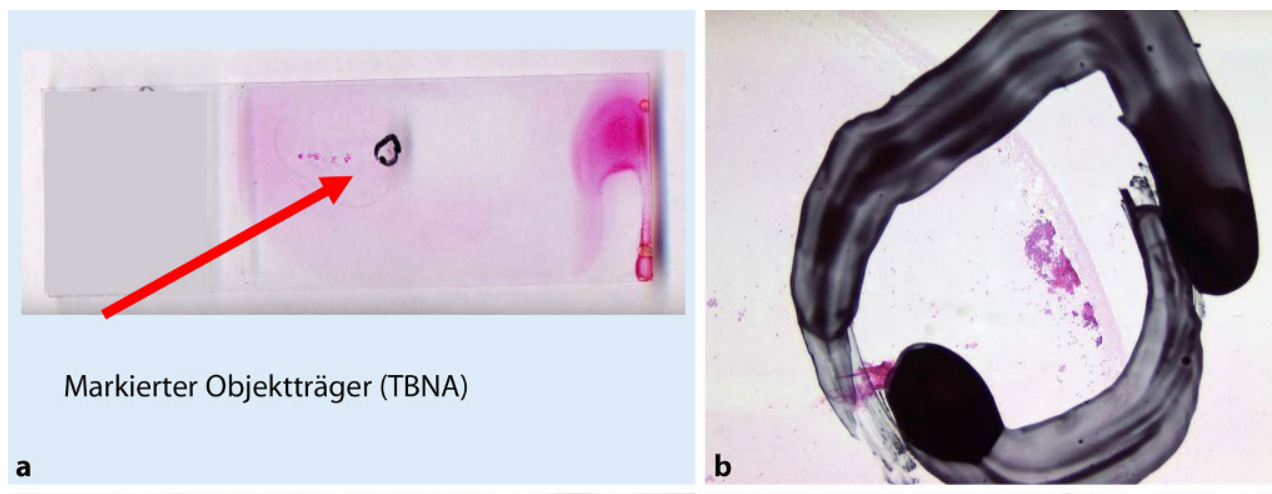

a
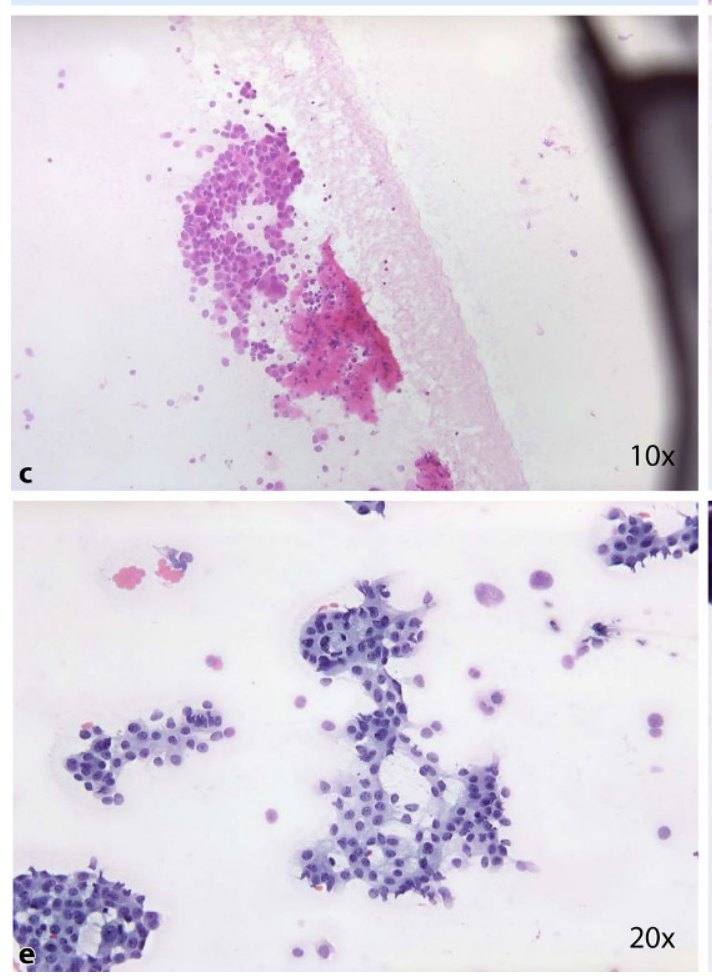

$2,5 x$

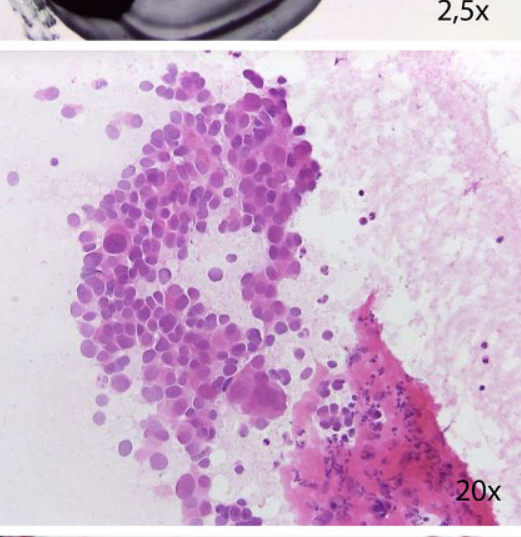

d

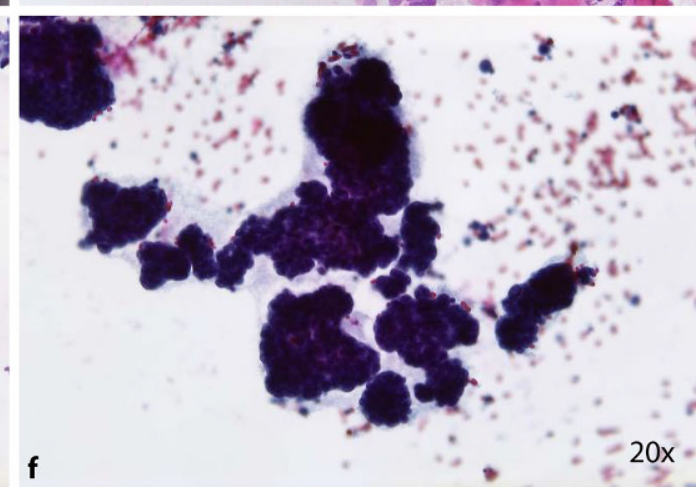

Abb. $4<$ a-d TBNA(transbronchiale Nadelaspiration)-Ausstrich mit Markierung für eine FISH(Fluoreszenz-in-situHybridisierung)-Untersuchung. Flächiger Tumorzellverband von mehr als hundert Karzinomzellen, die gut ausgebreitet sind. HE-Färbung. (b:Vergr. 2,5:1; c:Vergr. 10:1, d:Vergr. 20:1). e,f Sedimentausstrich eines Pleuraerguss, e Areal gut für die FISH geeignet. Die Zellen liegen gut ausgebreitet vor.f Areal gut für die DNA-Extraktion geeignet, aber wegen starker Überlagerung der Zellkerne nicht für FISH-Analysen. (๑ M. Engels) der Tumorzellen selber ist kein Problem (• Abb. 3).

Zytologische Proben können luftgetrocknet oder eingedeckelt beim Einzeichnen durch den Zytopathologen vorliegen. Daher kann die Markierung der Probe entweder auf der Folie/ Deckgläschen oder auf der Rückseite des Objektträgers vorkommen. Die Markierung wird dann, wie im Abschnitt Präanalytik beschrieben, übertragen. Falls von malignen Ergüssen ein Zellblock zur Verfügung steht, werden die molekularpathologischen Untersuchungen in der Regel am Zellblock durchgeführt [14]. Die Markierung der geeigneten Areale erfolgt genauso wie an einer Biopsie. Bei Bedarf können weitere Leerschnitte,
z.B. für die FISH-Analyse, angefertigt werden.

In unserer Institution ist die gleichzeitige Sequenzierung von 19 Genen und Genregionen in einem „Lungenpanel“ mithilfe der Parallelsequenzierung als Routinemethode etabliert [17]. Die isolierte DNA wird durch eine quantitative PCR (qPCR) auf ihre Amplifizierbarkeit und Konzentration hin vermessen. Sofern vorhanden, werden pro Primerpool 10 ng genomische DNA verwendet. Da das „Lungenpanel“ aus 4 Primerpools besteht, müssen insgesamt 40 ng DNA verwendet werden. Nach dem Resultat der Parallelsequenzierung und je nach Fragestellung schließen sich verschiedene FISH-Analysen an.

\section{Fluoreszenz-in situ-Hybridi- sierungen an zytologischen Präparaten}

Für eine FISH-Untersuchung braucht man im Präparat mindestens 100 maligne Zellen, die gut ausgebreitet liegen (- Abb. 4). Sie sollten möglichst ohne Überlagerung und gut von benignen Zellen abgrenzbar sein. Ein Vorteil der zytologischen Präparate für die FISHAnalyse sind die intakten Zellkerne, die nicht wie bei einem Gewebepräparat teilweise angeschnitten sind [18]. Die Auswertung der FISH-Analysen kann jedoch schwerer auszuwerten sein, da ein ganzer Zellkern bedeutet, dass man den Objektträger unter Feinfokussierung durchsehen muss. Diese Diagnostik ist 

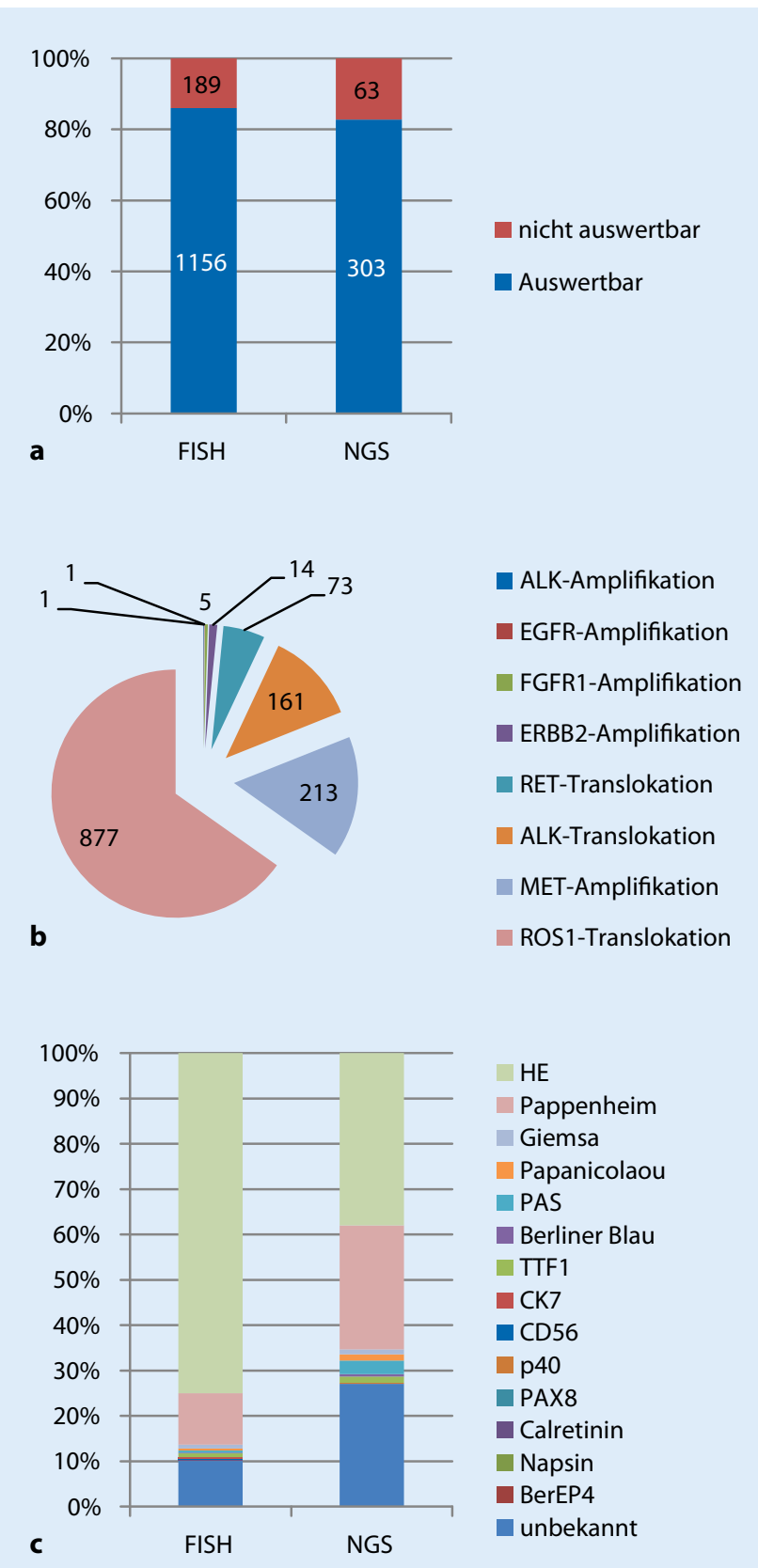

zeitintensiv, lohnt sich aber bei gering vorhandenem Material eines Patienten. Ein Problem bei den zytologischen Präparaten stellt die zurückgebliebene Färbung dar, die bei der Vorbehandlung zur Hybridisierung nicht komplett entfärbt werden kann. Rückstände können eine starke Autofluoreszenz im Hintergrund bilden, sodass die FISH-Analysen nicht auswertbar sein können.

Für jede FISH-Untersuchung ist ein solches Areal im Präparat mit 100 gut ausgebreiteten, gut abgrenzbaren Zellen
Abb. $5<$ a Anzahl FISH(Fluoreszenz-insitu-Hybridisierung)und NGS(Next Generation Sequencing)-Analysen an zytologischen Präparaten. b Diagramm der Gene und der Chromosomenaberration der durchgeführten FISH-Analysen. cFärbungen zytologischer Präparate, die für NGS- und FISH-Analysen weiterverwendet wurden. (@ M. Engels) der durchgeführten

Auswertung der molekularpathologischen Untersuchungen an zytologischen Präparaten

Im Zeitraum von 2016 bis Juli 2019 wurden in der Pathologie der Uniklinik Köln 1711 zytologische Präparate von Lungenkrebspatienten für eine molekularpathologische Diagnostik verwendet. Erfolgreich ausgewertet werden konnten 1459 Proben (85,3\%, • Abb. 5a). Zuvor wurde an den Präparaten die Diagnose morphologisch gesichert. Davon entfielen 1345 aufFISH- und 366 auf NGS(Next Generation Sequencing)-Untersuchungen. Es ließen sich $85,9 \%$ der NGS- und $82,2 \%$ der FISH-Analysen erfolgreich durchführen und auswerten (• Abb. 5a). Der überwiegende Teil $(65,2 \%)$ der FISH-Analysen waren ROS1-Translokationsuntersuchungen (• Abb. 5b). HEFärbungen wurden am häufigsten für die FISH-Analyse (75\%) und Parallelsequenzierung $(38 \%)$ verwendet. Die am zweithäufigsten verwendete Färbung war Pappenheim mit 11,3\% für die FISHAnalyse und 27,3\% für eine anschließende Parallelsequenzierung (• Abb.5c).

\section{Fazit für die Praxis}

- Molekularpathologische Diagnostik sollte an der Probe erfolgen, die dafür am besten geeignet ist (oft Biopsie aus Bronchus oder mediastinalem Lymphknoten).

- Es gibt aber einige Situationen, in denen es günstig ist, das zytologische Material zu benutzen:

- Die Biopsie war relativ klein.

- Die molekularpathologische Diagnostik wurde bei Erstdiagnose nicht durchgeführt und eine erneute Biopsie wird nicht gewünscht. nete Präparate vorhanden sind, nimmt man pro FISH-Untersuchung einen eigenen Objektträger. Bei zellreichen, qualitativ hochwertigen Ausstrichen können auch mehrere FISH-Analysen am selben Objektträger durchgeführt werden (• Abb. 2d-g). Gerade bei Sedimentausstrichen von Ergussflüssigkeit ist dies häufig möglich. 
- Falls ein maligner Erguss oder eine Metastase in einem peripheren, gut zugänglichen Lymphknoten vorliegt, ist es sinnvoll, die molekularpathologischen Untersuchungen dann aus leicht zugänglichem Material durchzuführen.

- Die gezeigten Erfolgsraten von $85,9 \%$ der Parallelsequenzierung und $82,2 \%$ der FISH(Fluoreszenzin-situ-Hybridisierung)-Analysen zeigen, dass unter bestimmten Voraussetzungen eine erneute $\mathrm{Bi}$ opsiegewinnung vermieden werden kann und dennoch die gewünschten Analysen mit hoher Erfolgswahrscheinlichkeit durchgeführt werden können.

\section{Korrespondenzadresse}

\section{Dr. rer. nat. Jana Fassunke}

Institut für Pathologie, Universitätsklinikum Köln

Kerpener Str. 62, 50924 Köln, Deutschland Jana.fassunke@uk-koeln.de

\section{Einhaltung ethischer Richtlinien}

Interessenkonflikt. J. Fassunke, M. Ball und M. Engels geben an, dass kein Interessenkonflikt besteht.

Alle beschriebenen Untersuchungen am Menschen oder an menschlichem Gewebe wurden mit Zustimmung der zuständigen Ethikkommission, im Einklang mit nationalem Recht sowie gemäß der Deklaration von Helsinki von 1975 (in der aktuellen, überarbeiteten Fassung) durchgeführt. Von allen beteiligten Patienten liegt eine Einverständniserklärung vor.

Open Access. Dieser Artikel wird unter der Creative Commons Namensnennung 4.0 International Lizenz veröffentlicht, welche die Nutzung, Vervielfältigung Bearbeitung, Verbreitung und Wiedergabe in jeglichem Medium und Format erlaubt, sofern Sie den/die ursprünglichen Autor(en) und die Quelle ordnungsgemäß nennen, einen Link zur Creative Commons Lizenz beifügen und angeben, ob Änderungen vorgenommen wurden.

Die in diesem Artikel enthaltenen Bilder und sonstiges Drittmaterial unterliegen ebenfalls der genannten Creative Commons Lizenz, sofern sich aus der Abbildungslegende nichts anderes ergibt. Sofern das betreffende Material nicht unter der genannten Creative Commons Lizenz steht und die betreffende Handlung nicht nach gesetzlichen Vorschriften erlaubt ist, ist für die oben aufgeführten Weiterverwendungen des Materials die Einwilligung des jeweiligen Rechteinhabers einzuholen.

Weitere Details zur Lizenz entnehmen Sie bitte der Lizenzinformation auf http://creativecommons.org/ licenses/by/4.0/deed.de.

\section{Literatur}

1. Pao W et al (2004) EGF receptor gene mutations are common in lung cancers from "never smokers" and are associated with sensitivity of tumors to gefitinib and erlotinib. Proc Natl Acad Sci U S A 101:13306-13311

2. Cardarella S et al (2013) Clinical, pathologic, and biologic features associated with BRAF mutations in non-small cell lung cancer. Clin Cancer Res 19:4532-4540

3. Soda M et al (2007) Identification of the transforming EML4-ALK fusion gene in non-small-cell lung cancer. Nature 448:561-566

4. Lin JJ, Shaw AT (2017) Recent advances in targeting ROS1 in lung cancer. J Thorac Oncol 12:1611-1625

5. Pisapia P et al (2017) ALK and ROS1 testing on lung cancer cytologic samples: perspectives. Cancer Cytopathol 125:817-830

6. Schildhaus HU et al (2015) MET amplification status in therapy-naive adeno- and squamous cell carcinomas of thelung. Clin Cancer Res 21:907-915

7. Li BT et al (2016) HER2 amplification and HER2 mutation are distinct molecular targets in lung cancers. JThorac Oncol 11:414-419

8. Nicholson AG, Geisinger K, Aisner SC et al (2015) Tumours of the lung- terminology and criteria in non-resection specimens. In: WHO (Hrsg) Classification of tumours of the lung, pleura, thymus and heart, 4. Aufl.

9. Bartlett JMS, Shaaban A, Schmitt F (2016) Molecular pathology with online resource: a practical guide for the surgical pathologist and cytopathologist

10. Baum JE et al (2017) Accuracy of next-generation sequencing for the identification of clinically relevant variants in cytology smears in lung adenocarcinoma. Cancer Cytopathol 125:398-406

11. Jain D et al (2017) Use of exfoliative specimens and fine-needle aspiration smears for mutation testing in lung adenocarcinoma. Acta Cytol 61:455-461

12. Yang GCH, Ali SZ (2012) Lung and mediastinum cytohistology

13. von MonacoSE, Khalbuss WE, PantanowitzL (2014) Endobronchial ultrasound-guided transbronchial needle aspiration (EBUS-TBNA): a practical approach

14. Collins GR, Thomas J, Joshi N, Zhang S (2012) The diagnostic value of cell block as an adjunct to liquid-based cytology of bronchial washing specimens in the diagnosis and subclassification of pulmonary neoplasms. Cancer Cytopathol 120:134-141

15. Herth FJ et al (2013) Diagnostic and predictive analyses of cytological specimens of non-small cell lung cancer: strategies and challenges. Pneumologie 67:198-204

16. da Cunha Santos G et al (2013) Minimizing delays in DNA retrieval: the "freezer method" for glass coverslip removal. Letter to the editor regarding comparative study of epidermal growth factor receptor mutation analysis on cytology smears and surgical pathology specimens from primary and metastatic lung carcinomas. Cancer Cytopathol 121:533

17. Hagmeyer L et al (2019) Bronchoscopic brushing from central lung cancer-next generation sequencing results are reliable. Lung 197:333-337

18. Savic S, Bubendorf $L$ (2012) Role of fluorescence in situ hybridization in lung cancer cytology. Acta Cytol 56:611-621
Interaktive Karte zur Medizininformatik

Wer treibt die Medizininformatik in Deutschland voran? Wo werden wegweisende IT-Lösungen entwickelt und klinisch erprobt, damit Ärzte Krankheiten zukünftig besser behandeln können?

Auf dem Weg in die Medizin der Zukunft spielt die Medizininformatik-Initiative des Bundesministeriums für Bildung und Forschung (BMBF) eine Schlüsselrolle. Vier Konsortien mit bundesweit mehr als 60 Partnern - darunter nahezu alle deutschen Universitätskliniken - bauen eine zukunftsweisende Infrastruktur auf: Datenintegrationszentren führen patientenbezogene Daten aus Forschung und Versorgung zusammen. Forschende analysieren diese Daten in enger Zusammenarbeit mit Medizinern. Das Ziel ist, IT-Lösungen zu entwickeln und zu erproben, mit deren Hilfe Ärzte Krankheiten künftig besser diagnostizieren und behandeln können. Mehr als 160 Millionen Euro stellt das BMBF von 2018 bis 2021 für diese Initiative bereit.

\section{Informationen für interessierte Laien}

Für interessierte Bürger hat der DLR-Projektträger im Auftrag des BMBF eine interaktive Karte entwickelt, die nun online verfügbar ist. Sie erläutert, wie die Initiative die Versorgung der Patienten konkret verbessern will - von einer präziseren Krebs- und Intensivmedizin bis hin zur Bekämpfung von Krankenhausinfektionen. Es können alle Standorte aufgerufen werden, die sich einem bestimmten Anwendungsfall widmen, beispielsweise auf dem Gebiet der Herz- oder Lungenerkrankungen (Herzinsuffizienz, Asthma, COPD) oder neurologischen Erkrankungen (Multiple Sklerose, Parkinson). Ein Mausklick auf einen Kartenpunkt - und es erscheinen detaillierte Informationen zum ausgewählten Standort und seiner Rolle in der Medizininformatik-Initiative.

Quelle: Bundesministerium für Bildung und Forschung (www.gesundheitsforschungbmbf.de) 